$6 \%$ rivaroxaban. Duration of anticoagulation could be ascertained in 28 patients: $43 \%$ had 6 months, 39\% lifelong, $7 \%$ had 4 months and the other 3 cases separately had 6 weeks, 2 months and 12 months.

Of the 51 patients who survived $>6$ months after diagnosis $59 \%$ had repeat CT imaging. The majority $(n=23)$ were anticoagulated and there was recanalisation in $61 \%$, partial recanalisation in $13 \%$ and cavernous transformation in $26 \%$. $83 \%$ of those with cavernous transformation had portal hypertension.

6 of the non-anticoagulated cases had a repeat CT. 1 had partial recanalisation and had developed varices. The other 5 had recanalisation but notably these cases were non-occlusive aPVT.

Conclusions This audit highlighted inconsistencies in the management of non-cirrhotic, non-malignant aPVT in our centre. On assessment of recanalisation several cases had cavernous transformation and resultant portal hypertension but many did not get this assessment and so their risk of portal hypertension is unknown.

As a result of these findings we are developing an aPVT pathway to guide clinicians, minimise complications of aPVT and develop a consistent approach in our trust.

\section{P220 FAILURE TO PERFORM REPEAT ASCITIC TAP AT 48HR HAS POORER OUTCOMES IN SPONTANEOUS BACTERIAL PERITONITIS}

${ }^{1}$ Jaclyn Yizhen Tan*, 'Jade King, 'Jennifer Ryan, 'Marsha Y Morgan, ${ }^{2,3}$ Emmanuel Q Wey, Rachel H Westbrook. 'UCL Institute of liver and digestive health, Division of Medicine, London, UK; ${ }^{2}$ Division of Infection and Immunity, University College London, London, UK; ${ }^{3}$ Department of Microbiology, Royal Free London, Centre for Clinical Microbiology, London, UK

\subsection{6/gutjnl-2020-bsgcampus.295}

Introduction In patients with Spontaneous Bacterial Peritonitis (SBP), acute kidney injury and high serum bilirubin are known predictors of in-hospital mortality. The effect of patient management on mortality is unknown. This study aims to identify predictors of in-hospital mortality, accounting for management of patients with SBP, according to EASL Clinical Practice Guidelines published in 2010.

Methods Clinico-demographic, biochemical and microbiological data from patients presenting between 2014 and 2019, with a first episode of SBP (ascitic fluid neutrophil count $\geq 250$ cell/ $\mathrm{cm}^{3}$ ) were reviewed. The primary endpoint was in-hospital mortality. Logistic regression was used to identify predictors of outcome.

Results Overall, 130 patients (median [IQR] age 58 [51 - 66] yr; 65\% male; aetiology: alcohol 36\%; MELD score 18 [13 25]) were included. Infection was nosocomial in 49\%; 35 had concomitant bacteraemia $(\mathrm{n}=14)$, respiratory $(\mathrm{n}=16)$ or urinary infections $(\mathrm{n}=9)$. Pathogens were identified in 57 (44\%) patients within 42 [36 - 50] hr post initial ascitic tap; antibiotic sensitivities were available by 53 [49 - 62] hr. Multidrug resistant pathogens (MDRP) were identified in 12 $(21 \%)$ of the $57 ; 10$ of the 12 showed $<25 \%$ reduction in ascitic neutrophil count at 48 hours.

There were $29(22.3 \%)$ in-hospital deaths; the median time to death was 6 [1 - 8] days. A total of 31 (24\%) patients were admitted to ITU and one-third $(\mathrm{n}=13)$ of this cohort died. One patient underwent liver transplantation. On univariate analysis, admission MELD, peripheral white cell count,
INR, serum creatinine, failure to culture a pathogen, failure to perform a 48-hour ascitic tap and development of acute kidney injury were predictors of in-hospital mortality. Age, nosocomial infection or the presence of a MDRP were not. Failure to perform a 48-hour ascitic tap (OR $[95 \% \mathrm{CI}]=11.2[2.9$ - 43.7], p < 0.01), acute kidney injury (9.1 [2.0 - 41.5], p $<0.01)$ and MELD score (1.2 [1.1 - 1.3], p < 0.01) retained significance on multivariate analysis.

Conclusions In-hospital mortality associated with SPB is unacceptably high at $22 \%$. Failure to repeat the ascitic tap at 48 hours, a recommendation based solely on expert opinion in the EASL guideline, was a highly significant prognostic factor allowing early identification of patients who fail to respond to empirical antibiotic therapy. This requirement should now become recommended practice.

\section{P221 NON-CIRRHOTIC VS CIRRHOTIC HCC: COMPARISON BETWEEN PATIENT CHARACTERISTICS, AETIOLOGY AND OUTCOMES}

${ }^{1}$ Jennifer Tham*, ${ }^{1}$ Tee Lin Goh, ${ }^{1}$ Stephen Barclay, ${ }^{2}$ Matthew Priest, ${ }^{1}$ Ewan Forrest, ${ }^{2}$ Andrew Fraser, ${ }^{1}$ Adrian Stanley. ${ }^{1}$ Glasgow Royal Infirmary, UK; ${ }^{2}$ Queen Elizabeth University Hospital, Glasgow, UK

\subsection{6/gutjnl-2020-bsgcampus.296}

Introduction Hepatocellular carcinoma (HCC) causes more than 5,400 deaths per year in the UK and is rising in incidence. Previously in the UK, HCC in non-cirrhotic livers was felt to be uncommon. We sought to establish the proportion of HCC occurring in patients without cirrhosis in our region of the UK and compare characteristics of those with cirrhosis.

Methods Data was collected from our prospectively collected database on patient demographics, liver aetiology, stage at presentation and outcome for patients diagnosed with HCC at our regional MDT from 2009 to 2015.

Results A total of 638 patients with HCC were included. 140 (21.9\%) had no underlying cirrhosis. Non-cirrhotic HCCs were older at diagnosis (72 years vs 68 years, $\mathrm{p}=0.001$ ), with a similar male to female ratio. Alcohol related liver disease (ArLD) was the most common underlying aetiology in patients with cirrhosis (59\%; see table 1), and along with Viral hepatitis was significantly more common than patients without cirrhosis. In contrast, unknown aetiology represented the majority of diagnoses, and was significantly greater in the non-cirrhotic cohort. Patients with non-cirrhotic HCC had more advanced malignant disease at diagnosis compared to cirrhotic HCC using Barcelona Clinic Liver Cancer (BCLC) staging, $\mathrm{p}<0.001$ (table 1). Liver transplant was performed in $4.2 \%$ of patients with cirrhotic HCC compared to no patients with non-cirrhotic HCC. Liver resection was performed in $4 \%$ cirrhotic versus 9\% non-cirrhotic. Radiofrequency ablation (RFA) was used in $7 \%$ and $1.4 \%$ of cirrhotic and non-cirrhotic HCC. Transarterial chemoembolization (TACE) was used in 25\% cirrhotic and 24\% non-cirrhotic HCC. Sorafenib was prescribed in 3\% cirrhosis and $6.4 \%$ non-cirrhotic HCC, with Sorafenib plus TACE used in $1 \%$ cirrhotic and $1.4 \%$ non-cirrhotic HCC. $59 \%$ and $57 \%$ of patients with cirrhosis and non-cirrhotic HCC, respectively, were treated with supportive care only. Median survival was lower in patients with cirrhotic HCC, 19.6 months, compared to non-cirrhotic HCC, 24.5 months, $\mathrm{p}=0.05$. 